\title{
Effects of SKF83959 on the excitability of hippocampal CA1 pyramidal neurons: a modeling study
}

\author{
Shang-lin ZHOU ${ }^{1,2, *}$, Hong-yuan $\mathrm{CHU}^{1,2}$, Guo-zhang $\mathrm{JIN}^{1}$, Jian-min $\mathrm{CUI}^{3}$, Xue-chu ZHEN ${ }^{1,3, *}$ \\ ${ }^{1}$ Department of Pharmacology II, Shanghai Institute of Materia Medica, Chinese Academy of Sciences, Shanghai 201203, China; \\ ${ }^{2}$ University of Chinese Academy of Sciences, Beijing 100049, China; ${ }^{3}$ Jiangsu Key Laboratory of Translational Research and Therapy \\ for Neuropsychiatric Disorders \& Department of Pharmacology, College of Pharmaceutical Sciences, Soochow University, Suzhou \\ 215123, China
}

Aim: 3-Methyl-6-chloro-7,8-hydroxy-1-(3-methylphenyl)-2,3,4,5-tetrahydro-1H-3-benzazepine (SKF83959) have been shown to affect several types of voltage-dependent channels in hippocampal pyramidal neurons. The aim of this study was to determine how modulation of a individual type of the channels by SKF83959 contributes to the overall excitability of CA1 pyramidal neurons during either direct current injections or synaptic activation.

Methods: Rat hippocampal slices were prepared. The kinetics of voltage-dependent $\mathrm{Na}^{+}$channels and neuronal excitability and depolarization block in CA1 pyramidal neurons were examined using whole-cell recording. A realistic mathematical model of hippocampal CA1 pyramidal neuron was used to simulate the effects of SKF83959 on neuronal excitability.

Results: SKF83959 (50 $\mu \mathrm{mol} / \mathrm{L})$ shifted the inactivation curve of $\mathrm{Na}^{+}$current by $10.3 \mathrm{mV}$ but had no effect on the activation curve in CA1 pyramidal neurons. The effects of SKF83959 on passive membrane properties, including a decreased input resistance and depolarized resting potential, predicted by our simulations were in agreement with the experimental data. The simulations showed that decreased excitability of the soma by SKF83959 (examined with current injection at the soma) was only observed when the membrane potential was compensated to the control levels, whereas the decreased dendritic excitability (examined with current injection at the dendrite) was found even without membrane potential compensation, which led to a decreased number of action potentials initiated at the soma. Moreover, SKF83959 significantly facilitated depolarization block in CA1 pyramidal neurons. SKF83959 decreased EPSP temporal summation and, of physiologically greater relevance, the synaptic-driven firing frequency.

Conclusion: SKF83959 decreased the excitability of CA1 pyramidal neurons even though the drug caused the membrane potential depolarization. The results may reveal a partial mechanism for the drug's anti-Parkinsonian effects and may also suggest that SKF83959 has a potential antiepileptic effect.

Keywords: SKF83959; hippocampus; pyramidal neuron; neuronal modeling; excitability; $\mathrm{Na}^{+}$channel; EPSP; temporal summation

Acta Pharmacologica Sinica (2014) 35: 738-751; doi: 10.1038/aps.2014.23; published online 26 May 2014

\section{Introduction}

SKF83959 [3-methyl-6-chloro-7,8-hydroxy-1-(3-methylphenyl)2,3,4,5-tetrahydro-1H-3-benzazepine], an agonist of the putative phosphatidylinositol (PI)-linked D1-like receptor, has drawn much attention for its unique pharmacological properties $^{[1-4]}$. It has been shown that SKF83959 produces potent antiparkinsonian effects in primate and rodent models ${ }^{[5-7]}$. Furthermore, SKF83959 has been reported to attenuate L-DOPA-

\footnotetext{
* To whom correspondence should be addressed.

E-mail zhoushanglin@gmail.com (Shang-lin ZHOU); zhenxuechu@suda.edu.cn (Xue-chu ZHEN)

Received 2013-11-26 Accepted 2014-03-03
}

induced dyskinesia (LID) in 6-OH-DOPA-lesioned rat models $^{[5,8]}$. Recently, SKF83959 has been shown to be an identified allosteric modulator of the sigma- 1 receptor and to have antidepressant effects ${ }^{[9]}$; moreover, SKF83959 has also been shown to improve the survival of pheochromocytoma cells (PC12 cells) subjected to $\mathrm{H}_{2} \mathrm{O}_{2}$ insult ${ }^{[10]}$. Both D1 receptor-dependent and -independent mechanisms have been suggested to be responsible for the range of the drug's effects ${ }^{[5-8,10]}$.

The passive membrane properties (input resistance, resting membrane potential, and other properties) and the excitability of neurons in the central neural system play fundamental roles in brain functions and diseases. For example, neuronal excitability and passive membrane properties in the entorhinal 
cortex have been shown to be significantly altered after 1 week following a single seizure episode ${ }^{[11]}$. It has also been shown that neuronal excitability is increased in the 6-OH-DOPAlesioned rat model of Parkinson's disease ${ }^{[12-14]}$. In our previous studies, we found that SKF83959 decreases input resistance and increases the membrane resting potential, and we showed that the compound also decreases the number of spikes evoked by the same current injection after compensating for the depolarization effect (setting the membrane potential the same as in control cells) ${ }^{[15]}$. It is known that many types of voltage-dependent ion channels (sodium channels, potassium channels, and others) contribute to the passive membrane properties and excitability of neurons. SKF83959 has been shown to regulate several types of these channels in the hippocampal pyramidal neuron ${ }^{[15-17]}$. First, SKF83959 (50 $\mu \mathrm{mol} / \mathrm{L})$ was found to decrease the amplitude of an $\mathrm{Na}^{+}$current by $67.6 \%{ }^{[17]}$, but how the drug modulates the activation and inactivation kinetics of $\mathrm{Na}^{+}$channels is unclear. Second, it was demonstrated that the drug exerts a potent inhibitory effect on the delayed rectifier $\mathrm{K}^{+}$current $\left(I_{\mathrm{K}}\right)$ and a slight effect on the A-type $\mathrm{K}^{+}$current $\left(I_{\mathrm{A}}\right)^{[16]}$. Third, SKF83959 was found to increase the amplitude of the hyperpolarization-activated current (HCN channel) and to shift the activation curve of $\mathrm{HCN}$ channels by $6 \mathrm{mV}^{[15]}$. It has been suggested that such actions of SKF83959 on voltage-dependent ion channels likely occur via direct interactions with the affected ion channels rather than indirectly through D1-like receptors because of the drug's fast onset of action. Intuitively, inhibition of sodium currents results in a decrease in neuronal excitability ${ }^{[18-20]}$; in contrast, a reduction of potassium currents may increase neuronal excitability in some conditions ${ }^{[21-24]}$. In regard to the HCN channel, the effect on neuronal excitability becomes more complex. The HCN channel alone could exert both excitatory (depolarizing the cell membrane) and inhibitory (decreasing input resistance) effects on neuronal excitability ${ }^{[25-27]}$. Because there has been only a limited number of pharmacological experiments and because the workings of these channels are complex, how the specific effect of SKF83959 on a single channel type contributes to the overall membrane excitability is not known. Moreover, neuronal excitability is also controlled by a summation of multiple excitatory postsynaptic potentials (EPSPs) in vivo. Considering that the HCN channel has a strong effect on EPSPs $^{[25,28,29]}$, how SKF83959 alters the synapse-driven firing (in which the neuron generates an action potential in response to a summation of EPSPs) of pyramidal neurons remains largely unknown.

Some types of neurons, such as pyramidal neurons in the hippocampus and dopaminergic neurons in the midbrain, cease firing when the stimulus is too strong; this phenomenon is known as depolarization block ${ }^{[30,31]}$. Depolarization block is regarded to have pathological relevance for some brain disorders, including epilepsy and schizophrenia ${ }^{[32-34]}$. Among all the mechanisms known to cause depolarization block, the inactivation of voltage-dependent $\mathrm{Na}^{+}$channels is believed to play a key role ${ }^{[31,35,36]}$. SKF83959 can decrease the amplitude of $\mathrm{Na}^{+}$currents ${ }^{[17]}$; however, whether the drug has an effect on the depolarization block of pyramidal neurons in the hippocampus remains to be determined.

In this study, we first examined the effects of SKF83959 on the gating kinetics of $\mathrm{Na}^{+}$channels using patch-clamp techniques in isolated, single hippocampal neurons. Using these and previously published experimental results, we refined a realistic mathematical model of the hippocampal CA1 pyramidal neuron that had been developed based on the morphology of actual pyramidal neurons and validated to reproduce important properties of these neurons ${ }^{[37]}$; we first used this mathematical model to explore how the hippocampal CA1 neuron may respond to stimuli (direct current injection or synapse-mediated EPSP) in the presence or absence of the effects of SKF83959. Next, we examined how the effects of SKF83959 on a single channel type may contribute to an overall change in neuronal excitability. The differences between the responses of the soma and those of the dendrite to SKF83959 were also considered. Finally, we conducted experiments that confirmed several of our modeling results.

\section{Materials and methods \\ Chemicals}

SKF83959 was synthesized in the Medicinal Chemistry Laboratory of the Shanghai Institute of Materia Medica, Chinese Academy of Sciences. All other chemicals were obtained from (Sigma-Aldrich Co, St Louis, MO, USA). SKF83959 was dissolved in dimethylsulfoxide (DMSO) and stored at $-20^{\circ} \mathrm{C}$. The stock solution was diluted to the desired concentration before use. DMSO at a final concentration of less than $0.1 \%$ had no detectable effect on the whole-cell experiments.

\section{Animals}

Sprague-Dawley rats were purchased from the Shanghai Laboratory Animal Co, LTD (Shanghai, China) and housed in plastic cages. Animals were allowed free access to a standard rodent diet and water. All experimental protocols were approved by the Institutional Animal Care and Use Committee of SIMM (Shanghai Institute of Materia Medica; protocol SIMM-2011-06-ZXC-07) and were in compliance with the Guidelines for the Care and Use of Laboratory Animals (National Research Council, People's Republic of China, 1996). All experiments were also conducted according to the National Research Council's guidelines.

\section{Electrophysiology experiments}

Experiments to assess the effects of SKF83959 on the gating kinetics of $\mathrm{Na}^{+}$channels were conducted on dissociated hippocampal neurons prepared from newborn (3-7 d) SpragueDawley rats as described previously ${ }^{[17,38]}$. Briefly, hippocampal slices (approximately $500 \mu \mathrm{m}$ ) were cut in oxygenated icecold dissociation solution containing $82 \mathrm{mmol} / \mathrm{L} \mathrm{Na}_{2} \mathrm{SO}_{4}, 30$ $\mathrm{mmol} / \mathrm{L} \mathrm{K}_{2} \mathrm{SO}_{4}, 5 \mathrm{mmol} / \mathrm{L} \mathrm{MgCl}_{2}, 10 \mathrm{mmol} / \mathrm{L} \mathrm{HEPES}$, and $10 \mathrm{mmol} / \mathrm{L}$ glucose; the $\mathrm{pH}$ of the dissociation solution was adjusted to 7.3 with $\mathrm{NaOH}$. The slices were then incubated in dissociation solution containing protease XXIII $(3 \mathrm{~g} / \mathrm{L})$ at $32^{\circ} \mathrm{C}$ for $8 \mathrm{~min}$. Next, the slices were placed in dissociation solution 
containing trypsin inhibitor type II-S $(1 \mathrm{~g} / \mathrm{L})$ and bovine serum albumin $(1 \mathrm{~g} / \mathrm{L})$ at room temperature; the solution was saturated with oxygen. Just before recording, the CA1 area was dissected out from several slices and triturated using a series of fire-polished Pasteur pipettes with decreasing tip diameters. The dissociated neurons were placed in a recording dish and superfused with oxygenated external solution containing $15 \mathrm{mmol} / \mathrm{L} \mathrm{NaCl}, 105 \mathrm{mmol} / \mathrm{L}$ Choline-Cl, $5 \mathrm{mmol} / \mathrm{L} \mathrm{KCl}$, $1 \mathrm{mmol} / \mathrm{L} \mathrm{CaCl}_{2}, 1.25 \mathrm{mmol} / \mathrm{L} \mathrm{MgCl}_{2}, 0.1 \mathrm{mmol} / \mathrm{L} \mathrm{CdCl}_{2}$, $10 \mathrm{mmol} / \mathrm{L}$ HEPES, $20 \mathrm{mmol} / \mathrm{L} \mathrm{TEA}-\mathrm{Cl} 20$, and $10 \mathrm{mmol} / \mathrm{L}$ glucose, with the $\mathrm{pH}$ adjusted to 7.4 with $\mathrm{NaOH}$. Whole-cell voltage-clamp recordings were made from large pyramidalshaped neurons using an Axopatch 200A amplifier (Molecular Devices, Sunnyvale, CA, USA) at room temperature. Recording electrodes (resistance of 4-5 M 2 ) were pulled from borosilicate glass pipettes with a Flaming/Brown micropipette puller (Model P-97, Sutter Instrument, Novato, CA, USA) and were filled with intra-pipette fluid containing $130 \mathrm{mmol} / \mathrm{L}$ $\mathrm{CsCl}, 10 \mathrm{mmol} / \mathrm{L} \mathrm{NaCl}, 1 \mathrm{mmol} / \mathrm{L} \mathrm{CaCl}_{2}, 10 \mathrm{mmol} / \mathrm{L}$ HEPES, $1 \mathrm{mmol} / \mathrm{L} \mathrm{MgCl}_{2}$, and $10 \mathrm{mmol} / \mathrm{L}$ EGTA, with $\mathrm{pH}$ adjusted to 7.4 with $\mathrm{KOH}$. A voltage-activated $\mathrm{Na}^{+}$current was elicited by a series of command protocols provided in pClamp 9.2 software via a Data-1322A interface (Molecular Devices, Sunnyvale, CA, USA). Series resistance was compensated $75 \%-85 \%$. Linear leak and residual capacitance were subtracted online using a $\mathrm{P} / 4$ protocol. Signals were filtered at $2-10 \mathrm{kHz}$ and sampled at frequencies of $10-40 \mathrm{kHz}$ via a Data-1322A interface (Molecular Devices, Sunnyvale, CA, USA) and stored in an IBM-compatible computer.

Experiments studying excitability and depolarization block at the soma were performed on pyramidal neurons in the hippocampal CA1 area of the brain slices of Sprague-Dawley rats (2-3 weeks old) using whole cell recording. Briefly, rats were anesthetized with $20 \%$ chloral hydrate and then decapitated. The brains were rapidly transferred into chilled and oxygenated artificial cerebrospinal fluid (ACSF) containing $119 \mathrm{mmol} / \mathrm{L} \mathrm{NaCl}, 3 \mathrm{mmol} / \mathrm{L} \mathrm{KCl}, 1.3 \mathrm{mmol} / \mathrm{L} \mathrm{MgCl}_{2}, 2.5$ $\mathrm{mmol} / \mathrm{L} \mathrm{CaCl}, 1 \mathrm{mmol} / \mathrm{L} \mathrm{NaH}_{2} \mathrm{PO}_{4}, 11 \mathrm{mmol} / \mathrm{L}$ glucose, and $26 \mathrm{mmol} / \mathrm{L} \mathrm{NaHCO}_{3}$. Hippocampal slices $(350 \mu \mathrm{m})$ were cut using an M752 vibroslice (Campden Instruments Ltd, Loughborough, UK) and incubated in the $37^{\circ} \mathrm{C}$ oxygenated ACSF for at least $1 \mathrm{~h}$. The slices were then transferred to a chamber filled with $31^{\circ} \mathrm{C}$ oxygenated ACSF for electrophysiological recordings. Whole-cell recordings were performed under a DIC upright microscope (BX51WI, Olympus, Tokyo, Japan) using a MultiClamp 700A amplifier (Molecular Devices, Sunnyvale, CA, USA). Recording electrodes (resistance of 4-5 M 2 ) were pulled from borosilicate glass pipettes with a Flaming/Brown micropipette puller (Model P-97, Sutter Instrument, Novato, CA, USA) and were filled with intrapipette fluid containing $140 \mathrm{mmol} / \mathrm{L} \mathrm{K}$-gluconate, $1 \mathrm{mmol} / \mathrm{L}$ $\mathrm{MgCl}_{2}, 10 \mathrm{mmol} / \mathrm{L}$ HEPES, $2 \mathrm{mmol} / \mathrm{L}$ ATPNa, $0.1 \mathrm{mmol} / \mathrm{L}$ $\mathrm{GTPNa}_{3}$, and $0.05 \mathrm{mmol} / \mathrm{L}$ EGTA, with $\mathrm{pH}$ adjusted to 7.25 with $\mathrm{KOH}$. Data with a series resistance (15-20 M $\Omega$ ) change $>20 \%$ were discarded. Data acquisition and analysis were performed using a digitizer (DigiData 1322A, Molecular Devices,
Sunnyvale, CA, USA) and pClamp 9.2 software. Signals were filtered at $2 \mathrm{kHz}$ and sampled at $10 \mathrm{kHz}$. The liquid junction potential of approximately $10 \mathrm{mV}$ was not corrected. Drugs were delivered by perfusion. Picrotoxin $(100 \mu \mathrm{mol} / \mathrm{L}), \mathrm{CNQX}$ $(10 \mu \mathrm{mol} / \mathrm{L})$ and APV $(50 \mu \mathrm{mol} / \mathrm{L})$ were present in the solution at all times to block synaptic transmission.

\section{Modeling}

All modeling work was carried out using the NEURON simulation software ${ }^{[39]}$. A previously published realistic model of a hippocampal CA1 pyramidal neuron ${ }^{[37]}$, which was based on the work of Migliore et al (1999) ${ }^{[40]}$, was modified for use in this study. Briefly, all the passive properties of the modified model, including the membrane resistance ( $\mathrm{Rm})$, the membrane capacitance $(\mathrm{Cm})$, the axial resistance and other properties, remained the same as in the previously published mode ${ }^{[37]}$. Several active channel mechanisms were implemented in the model, including a voltage dependent sodium channel (na), a delayed rectifier potassium channel ( $k d r)$, an A-type potassium channel (ka) and an HCN channel (h). The maximum conductance of each channel mechanism remained the same in the new model as in the original model, with the exception that the conductance of the kdr channel was set to $0.006 \mathrm{~S} / \mathrm{cm}^{2}$. The maximum conductance of the HCN channel increased linearly along the apical dendrite from the soma, and consequently, the HCN channel density in the apical dendrite was much higher than that in soma. To observe the exact effect of SKF83959 on the membrane potential, we did not balance the passive reversal potential in accordance with currents of the na, kdr or HCN channels, as previously described ${ }^{[37]}$, and as a consequence, the resting membrane potential was more positive than the passive reversal potential because of the opening of $\mathrm{HCN}$ channels at resting potential.

To simulate the amplitude of a given current, we set the maximum conductance of the other channels to zero and elicited the current using different voltage clamp protocols. The input resistance was calculated as the steady state membrane potential change in response to a $-50 \mathrm{pA}$ direct current injection at the soma divided by $-50 \mathrm{pA}$. The voltage sag ratio caused by the opening of the HCN channel was defined as the ratio of the peak voltage deflection to the steady-state voltage deflection. The rheobase current was measured as the minimal current required to be injected to elicit a single action potential during $500 \mathrm{~ms}$ (with a testing step of $1 \mathrm{pA}$ ). The action potential threshold was defined as the membrane potential at which $\mathrm{d} V / \mathrm{d} t$ crosses $20 \mathrm{mV} / \mathrm{ms}$ with rheobase current injection.

The depolarization block threshold was measured as the minimal current required to induce a block of firing during $1 \mathrm{~s}$ with the membrane potential compensated to the control level. All current clamp or voltage clamp simulations were conducted using the IClamp and SEClamp objects of NEURON ${ }^{[39]}$.

The effect of SKF83959 $(50 \mu \mathrm{mol} / \mathrm{L})$ on each channel was modeled by changing the maximum conductance alone or in combination with changing the half voltage of activation (or inactivation) of each channel in accordance with our experimental results. 
Modeling the synapse involved both AMPA- and NMDAlike synaptic conductances ${ }^{[41]}$. The rise and fall time constants for AMPA receptors were $0.5 \mathrm{~ms}$ and $3 \mathrm{~ms}$ respectively and for NMDA receptors were $2.3 \mathrm{~ms}$ and $100 \mathrm{~ms}$ respectively ${ }^{[42]}$. The maximum NMDA conductance $\left(G_{\max }\right.$ NMDA) was set to one third of that of AMPA $\left(G_{\text {max }} \text { AMPA) }\right)^{[42]}$ (see below). For the EPSP temporal summation simulation, one synapse was placed on one apical dendrite $210 \mu \mathrm{m}$ from the soma, and the

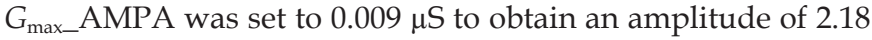
$\mathrm{mV}$ for the first EPSP. For the EPSP-spike coupling simulation, the synapse was modeled the same as for the EPSP temporal summation except that a series of varied $G_{\max }$ AMPA were tested. For the synapse-driving firing simulation, five independent synapses (with a $G_{\max }$ AMPA of $0.021 \mu \mathrm{S}$ ) were randomly placed on the apical dendritic compartments 150$400 \mu \mathrm{m}$ from the soma and randomly (Poisson) activated at different average frequencies (EPSP frequencies). Each average firing rate (synapse-driving firing frequency) of a pyramidal neuron in response to a given average EPSP frequency was calculated from 20 simulations of $1 \mathrm{~s}$ duration ${ }^{[37,43]}$.

In some experiments and simulations, the membrane potential was compensated to the control level by direct injecting a negative current at the site where the recording was being made, using a current clamp to hyperpolarize the membrane potential to the value of that for control.

\section{Data analysis}

The steady-state activation and inactivation curve for $\mathrm{Na}^{+}$ channels was fitted using a single-exponential Boltzmann equation:

$$
\begin{aligned}
& G / G_{\max }=1 /\left\{1+\exp \left[\left(V-V_{1 / 2}\right) / k\right]\right\}, \text { with } \\
& G=I /\left(V-V_{\mathrm{e}}\right),
\end{aligned}
$$

where $G$ is the membrane conductance, $G_{\max }$ is the maximum membrane conductance, $I$ is the $\mathrm{Na}^{+}$current recorded as the voltage varies, $V$ is the membrane potential, $V_{1 / 2}$ is the potential at which the value of $G$ is half that of $G_{\max }, V_{\mathrm{e}}$ is the reversal potential of $\mathrm{Na}^{+}$and $k$ is the slope of the curve.

EPSP temporal summation was calculated as follows:

EPSP summation $=\left(V \_5\right.$ thEPSP $-V \_1$ stEPSP $) /\left(V \_1\right.$ stEPSP $\left.-V m\right)$, where $V \_5$ thEPSP is the peak membrane potential at the fifth EPSP, $V \_1 s t E P S P$ is the peak membrane potential at the first EPSP and $V \mathrm{~m}$ is the resting membrane potential.

Data are presented as the mean \pm SEM. Statistical significance was assessed by paired Student's $t$-test, and $P<0.05$ was considered significant. Data analyses were performed using GraphPad Prism software Version 5.0 (San Diego, USA).

\section{Results}

The effects of SKF83959 on the gating kinetics of $\mathrm{Na}^{+}$channels In our previous work, we found that SKF83959 (50 $\mu \mathrm{mol} / \mathrm{L})$ significantly decreased the amplitude of $\mathrm{Na}^{+}$currents in hippocampal pyramidal neurons ${ }^{[17]}$. Here, we tested whether SKF83959 could modify the activation or inactivation curve of $\mathrm{Na}^{+}$channels in dissociated hippocampal neurons. As shown in Figure 1A, SKF83959 (50 $\mu \mathrm{mol} / \mathrm{L})$ did not significantly alter the activation curve of $\mathrm{Na}^{+}$channels; $V_{1 / 2}$ of the activa-
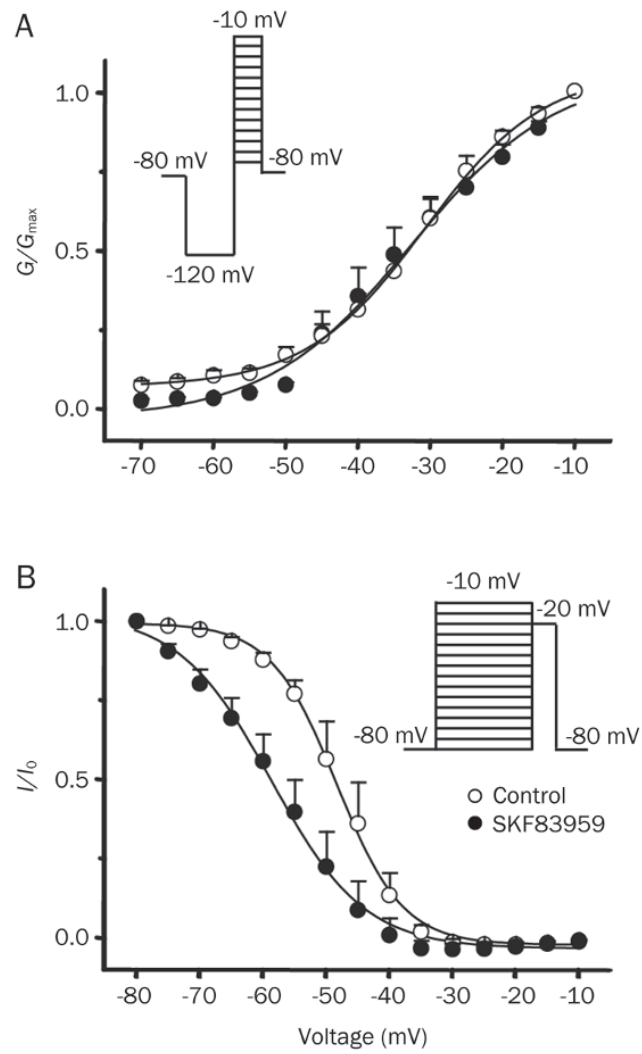

Figure 1. Effects of SKF83959 on the gating kinetics of the $\mathrm{Na}^{+}$channel in dissociated hippocampal neurons experiments. (A) Activation curve of the voltage-dependent $\mathrm{Na}^{+}$channel. The inset shows the voltageclamping protocol; the membrane potential was clamped at $-80 \mathrm{mV}$, and after a prepulse $(-120 \mathrm{mV}, 50 \mathrm{~ms})$, a series of voltages, from $-70 \mathrm{mV}$ to $-10 \mathrm{mV}$ with steps of $5 \mathrm{mV}$ and of $30 \mathrm{~ms}$ duration, were applied to the cell. Each $\mathrm{Na}^{+}$current was converted to conductance. $G_{\text {max }}$ : the maximum conductance. Mean \pm SEM $(n=7)$. (B) Inactivation curve of voltagedependent $\mathrm{Na}^{+}$channel. The inset shows the voltage-clamping protocol; the membrane potential was clamped at $-80 \mathrm{mV}$, and a series of voltages, from $-80 \mathrm{mV}$ to $-10 \mathrm{mV}$ with steps of $5 \mathrm{mV}$ and of $100 \mathrm{~ms}$ duration, were applied to induce the inactivation of $\mathrm{Na}^{+}$channels. The voltage was then clamped at $-20 \mathrm{mV}$ for $30 \mathrm{~ms}$, and $\mathrm{Na}^{+}$currents $(I)$ were measured. $I_{0}$, the maximum current. Mean \pm SEM $(n=5)$. Control: open circle. SKF83959: black circle.

tion curve was $-33.4 \pm 3.5 \mathrm{mV}$ before treatment with SKF83959 and $-31.6 \pm 2.0 \mathrm{mV}(n=7, P>0.05)$ after treatment. The slope of the activation curve was $10.0 \pm 0.7$ before treatment and $8.9 \pm 0.4$ after treatment $(n=7, P>0.05)$. In contrast, SKF83959 (50 $\mu \mathrm{mol} / \mathrm{L})$ significantly shifted $V_{1 / 2}$ of the inactivation curve from $-48.1 \pm 2.0 \mathrm{mV}$ before SKF83959 application to a more hyperpolarized level of $-58.4 \pm 2.8 \mathrm{mV}(n=5, P<0.01)$. The slope of the inactivation curve was $5.2 \pm 0.2$ before treatment and $7.4 \pm 0.6$ after treatment $(n=5, P<0.01$, Figure $1 \mathrm{~B})$.

Modeling the effects of SKF83959 on $\mathrm{Na}^{+}$, kdr and $\mathrm{HCN}$ channels Using a previously published realistic model of the hippocampal CA1 pyramidal neuron ${ }^{[37,40]}$ as a starting point, we selectively modified several parameters of the ion channel mecha- 
nisms of the model to simulate the major effects of SKF83959 $(50 \mu \mathrm{mol} / \mathrm{L})$ observed in experiments. First, to reproduce in our model the experimental result that SKF83959 $(50 \mu \mathrm{mol} / \mathrm{L})$ decreased the amplitude of the $\mathrm{Na}^{+}$current to $33.4 \%$ of that of the contro ${ }^{[17]}$, we first decreased the maximum conductance of $\mathrm{Na}^{+}$channels from $0.032 \mathrm{~S} / \mathrm{cm}^{2}$ to $0.023 \mathrm{~S} / \mathrm{cm}^{2}$ and shifted the $V_{1 / 2}$ of the inactivation curve down by $10.3 \mathrm{mV}$, as described above. The $\mathrm{Na}^{+}$current amplitude observed with SKF83959 treatment was then reduced to $33.3 \%$ of that of the control (Figure 2A). Second, we decreased the maximum conductance of $\mathrm{kdr}$ channels from $0.006 \mathrm{~S} / \mathrm{cm}^{2}$ to $0.003 \mathrm{~S} / \mathrm{cm}^{2}$, which changed the amplitude of the kdr current to $50 \%$ of that of the control, in accordance with previous experimental results ${ }^{[16]}$ (Figure 2B). Third, we increased the maximal conductance of $\mathrm{HCN}$ channels from $0.0006 \mathrm{~S} / \mathrm{cm}^{2}$ to $0.00092 \mathrm{~S} / \mathrm{cm}^{2}$ and shifted the $\mathrm{HCN}$ channel activation curve by $6 \mathrm{mV}$. As illustrated in Figure $2 \mathrm{C}$, the amplitude of the $\mathrm{HCN}$ channel current, measured at $-105 \mathrm{mV}$, was increased by $54.7 \%$, which was consistent with experimental data ${ }^{[15]}$. Thus, we have successfully reproduced previously published experimental results using our mathematical model.

A

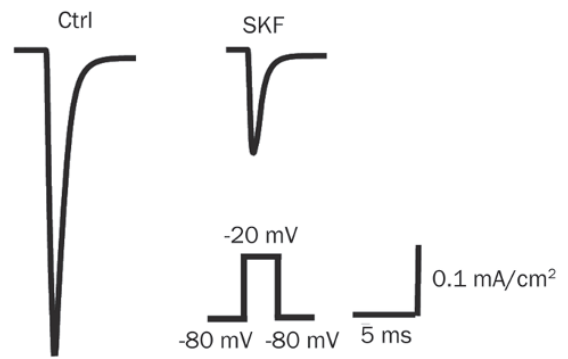

B
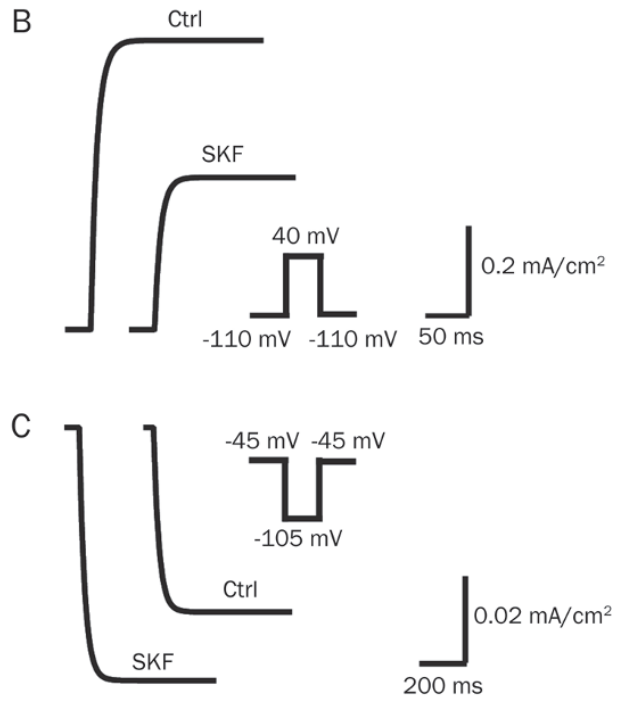

Figure 2. Modeling the effects of SKF83959 on $\mathrm{Na}^{+}, \mathrm{kdr}$, and $\mathrm{HCN}$ channels. The effects of SKF83959 (50 $\mu \mathrm{mol} / \mathrm{L})$ on $\mathrm{Na}^{+}, \mathrm{kdr}$, and $\mathrm{HCN}$ channels were modeled as described. (A) The effects of SKF83959 on $\mathrm{Na}^{+}$current. (B) The effects of SKF83959 on kdr current. (C) The effects of SKF83959 on HCN channel current. Ctrl, control; SKF, SKF83959; insets, voltage-clamping protocols.
Modeling the effects of SKF83959 on the passive membrane properties of the hippocampal pyramidal neuron

We first evaluated the effects of SKF83959 on the passive properties of hippocampal pyramical neurons using our mathematical model of the hippocampal pyramidal neuron. As shown in Figure 3, our model predicts that application of SKF83959 depolarizes the membrane potential by $4.1 \mathrm{mV}$. This depolarization appears to be primarily attributable to the increase in $\mathrm{HCN}$ current because modifying the $\mathrm{HCN}$ channel alone caused a $4 \mathrm{mV}$ depolarization, whereas altering kdr or $\mathrm{Na}^{+}$ channels resulted in either a $0.05 \mathrm{mV}$ or a $0 \mathrm{mV}$ depolarization, respectively (Figure 3D). The voltage sag ratio was increased from 1.16 to 1.32 when the resting membrane potential was
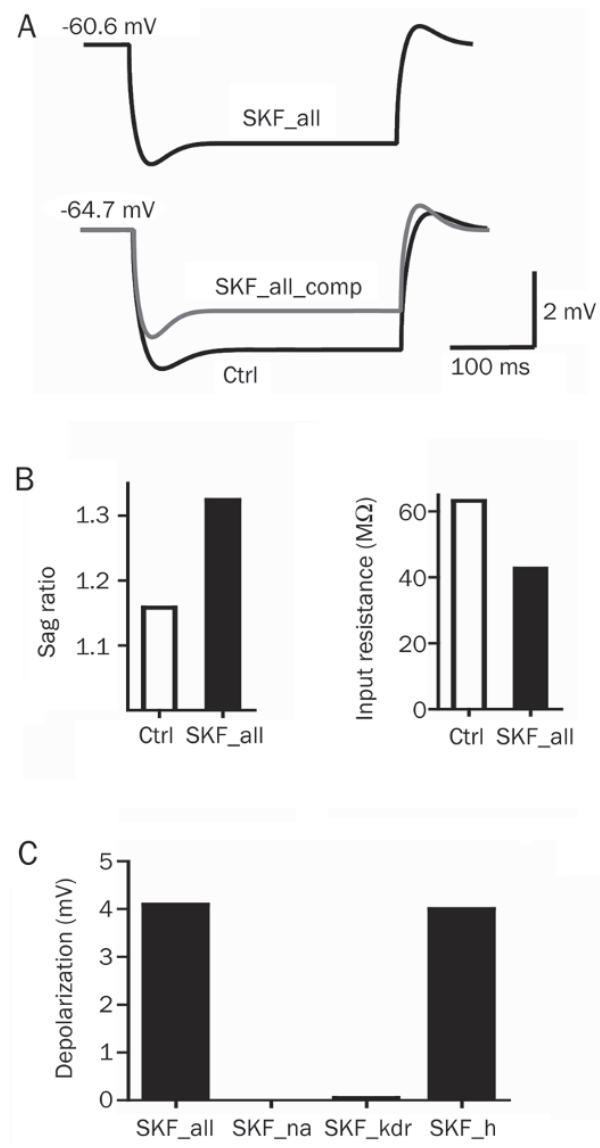

Figure 3. Effects of SKF83959 on the passive membrane properties of the hippocampal pyramidal neuron in simulations. (A) Membrane potential responses of the hippocampal pyramidal neuron to a $-50 \mathrm{pA}$, $300 \mathrm{~ms}$ pulse for control and SKF83959 treated cells with (gray trace) or without compensating membrane potential to the control level (top) as described in the Materials and methods. (B) Effects of SKF83959 on the sag ratio with membrane potential compensation measured as described in the Materials and methods. (C) Effects of SKF83959 on the input resistance. (D) Effects of SKF83959 on the membrane potential. Ctrl, control; SKF_all, overall effects of SKF83959 without membrane potential compensation; SKF_all_comp, overall effects of SKF83959 with membrane potential compensation; SKF_na, modifying $\mathrm{Na}^{+}$channels alone; SKF_kdr, modifying kdr channels alone; SKF_h, modifying HCN channels alone. 
compensated back to the control level by direct current injection at the soma (-82 pA, Figure $3 \mathrm{~A}$ and $3 \mathrm{~B})$. Moreover, the input resistance decreased from 63.2 to $42.6 \mathrm{M} \Omega$ (Figure 3C). All of these effects of SKF83959 on passive membrane properties were similar to experimental results ${ }^{[15]}$.

\section{Effects of SKF83959 on excitability of the soma in hippocampal pyramidal neurons}

To determine the effects of SKF83959 on soma excitability in hippocampal pyramidal neurons, we elicited trains of action potentials by direct current injection (steps with 25 pA increments) at the soma. When the membrane potential was compensated to the control level, modifying all three channels (with SKF83959, as described above) decreased the number of action potentials from 13 for control to 1 for SKF83959 treated neurons, with 350 current injections (Figure 4A, B \&C). However, when the depolarized membrane potential was not compensated, SKF83959 was able to increase the excitability of the soma, as 15 spikes were detected. To further test the contribution of a single channel type to this excitability, we modified one channel type at a time, leaving all others unchanged. Modifying the $\mathrm{Na}^{+}$channel alone decreased the number of spikes from 8 to 1 (elicited by $300 \mathrm{pA}$ current injection, Figure $4 \mathrm{~A} \& \mathrm{~B})$. However, modifying the $\mathrm{kdr}$ channel alone increased the number of spikes from 13 to 15 , regardless of whether the membrane potential was compensated (Figure $4 \mathrm{~A}-4 \mathrm{C}$ ). As for the HCN channel, modifying its current alone decreased the spike number to 4 when the membrane potential was compensated, but increased the spike number to 15 when the membrane potential was not compensated (current injection: $350 \mathrm{pA}$, Figure 4A-4C). These results were further confirmed by whole-cell recordings in the hippocampal CA1 area, which showed that the number of spikes after SKF83959 $(50 \mu \mathrm{mol} / \mathrm{L})$ treatment significantly increased, from $6.4 \pm 0.6$ to $7.7 \pm 0.7$, without compensating membrane potential to control level $(n=7$, $P<0.05$ ), but significantly decreased to $4.0 \pm 0.7$ with membrane potential compensation $(n=7, P<0.001,75 \mathrm{pA}$ current injection, Figure 4D and 4E).

We then measured the rheobase current and spike threshold with the membrane potential compensated to the control level. As shown in Figure 4F, SKF83959 increased the rheobase current by $67 \mathrm{pA}$. Modifying the $\mathrm{Na}^{+}$or $\mathrm{HCN}$ channel alone also increased the rheobase current by $17 \mathrm{pA}$ and $63 \mathrm{pA}$, respectively. In contrast, modifying the $\mathrm{kdr}$ channel decreased the rheobase current by $13 \mathrm{pA}$. The spike threshold was $-39 \mathrm{mV}$ for the control condition, but SKF83959 increased the spike threshold to $-37.5 \mathrm{mV}$ (Figure 4G). This effect was most likely due to the inhibition of $\mathrm{Na}^{+}$channels because we found that modifying the $\mathrm{Na}^{+}$channel alone increased the threshold to $-37.1 \mathrm{mV}$, whereas modifying the other two channels resulted in only a slight change in the threshold (to $-39.3 \mathrm{mV}$ for the $\mathrm{kdr}$ channel, $-39 \mathrm{mV}$ for the HCN channel, Figure 4G). All these results showed that SKF83959 decreased the excitability of the soma only when the membrane potential was compensated to the control level.

\section{Effects of SKF83959 on dendritic excitability in hippocampal pyramidal neurons}

Dendrites of the pyramidal neuron are very different from the soma in many ways, including in their passive properties and ion channel densities. We next recorded trains of action potential simultaneously at one apical dendrite $(210 \mu \mathrm{m}$ from the soma) and at the soma in response to direct current injection at this dendrite. SKF83959 also depolarized the membrane in dendrites, from -63.8 to $-59.4 \mathrm{mV}$. However, in contrast with the results shown in Figure 4B\&C, SKF83959 decreased the spike number detected in the dendrite and soma even when the membrane potential was not compensated (11 spikes for control cells compared with 6 spikes for SKF83959 treated cells, $550 \mathrm{pA}$ current injection, Figure 5). Modifying the $\mathrm{Na}^{+}$ channel alone decreased the spike number at every tested current level (Figure 5B, 5C). Similar to the results shown in Figure 4, modifying kdr channels alone increased the number of spikes without membrane potential compensation (Figure $5)$. On the other hand, modifying the HCN channel alone resulted in almost the same number of spikes as in control cells when membrane potential was not compensated (Figure 5). In contrast with results obtained at the soma, SKF83959 slightly decreased the spike threshold from $-24.7 \mathrm{mV}$ to -25.8 $\mathrm{mV}$, and modifying $\mathrm{Na}^{+}$channels alone slightly increased the threshold to $-24.2 \mathrm{mV}$. In comparison, modifying kdr or HCN channels decreased the spike threshold to $-25.3 \mathrm{mV}$ and -25.1 $\mathrm{mV}$, respectively (Figure 5D). However, the rheobase current scenario was similar to that at soma. SKF83959 increased the rheobase current from $491 \mathrm{pA}$ to $641 \mathrm{pA}$. Individually modifying $\mathrm{Na}^{+}, \mathrm{kdr}$, or $\mathrm{HCN}$ channels differentially altered the rheobase current: $566 \mathrm{pA}$ for $\mathrm{Na}^{+}, 476 \mathrm{pA}$ for $\mathrm{kdr}$ and $602 \mathrm{pA}$ for HCN (Figure 5E). Taken together, these results suggest that SKF83959 decreases dendritic excitability even without membrane potential compensation and that inhibition of the $\mathrm{Na}^{+}$channel likely plays a major role in the drug's effect on dendrites.

\section{SKF83959 facilitates depolarization block in hippocampal pyramidal neurons both in simulations and experiments}

Depolarization block represents a state in which neurons stop firing because the current injected has reached a relatively high level. Modeling studies have suggested that depolarization block could happen in vivo, and it has been suggested that depolarization block could play an important role in some pathological conditions, such as epilepsy ${ }^{[31]}$. Because both $\mathrm{Na}^{+}$ and kdr channels affect depolarization block, we examined the effects of SKF83959 on the amplitude of the minimal current required to induce depolarization block by current injection at the soma, while compensating the membrane potential to the control level. According to our modeling results we found that SKF83959 markedly decreased the threshold for the induction of depolarization block (1.29 nA for control cells and $0.59 \mathrm{nA}$ for SKF83959 treated cells, Figure 6). Specifically, modifying the $\mathrm{Na}^{+}$or kdr channels alone decreased the threshold to $0.68 \mathrm{nA}$ and $0.9 \mathrm{nA}$, respectively. In contrast, modifying 
A

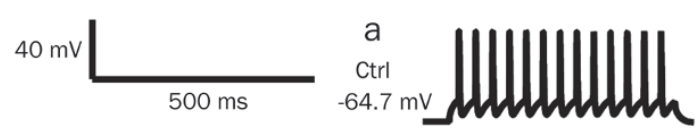

b
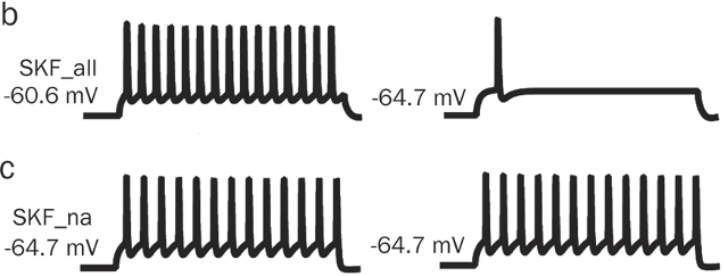

||l|||||

d

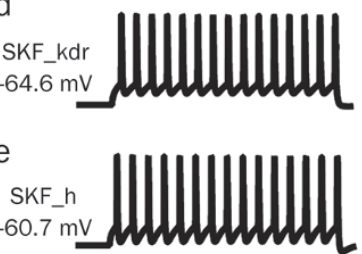

Without comp
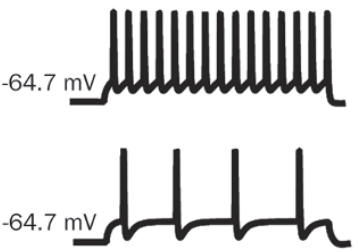

With comp
D
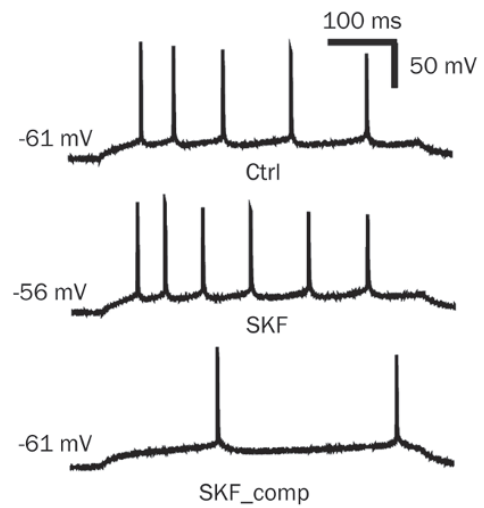

F

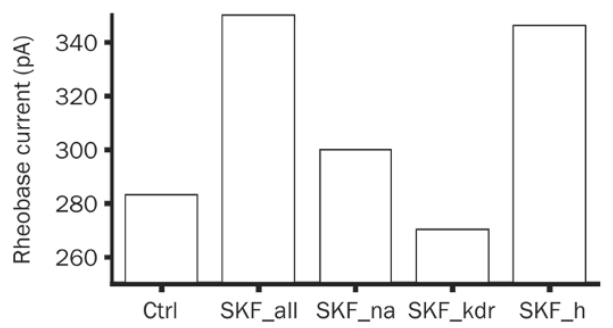

B

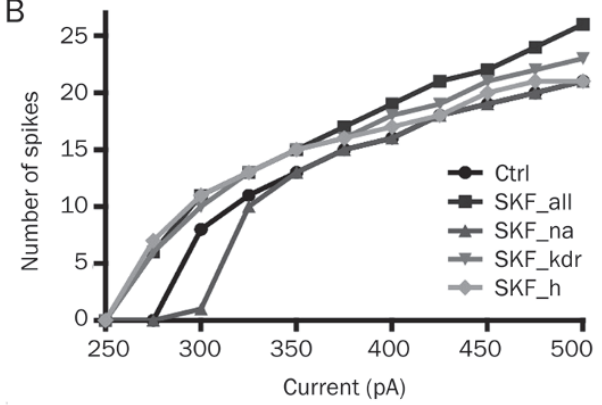

C

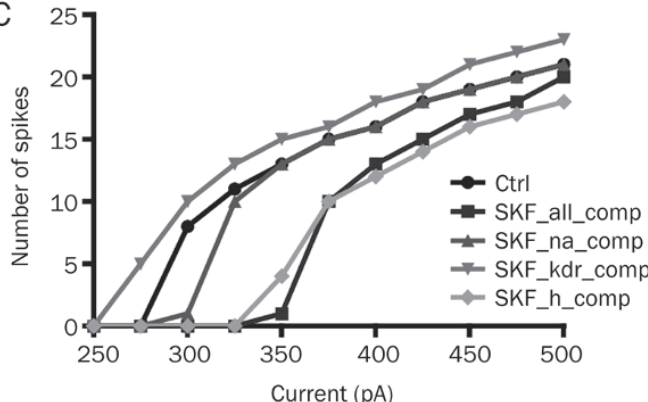

E

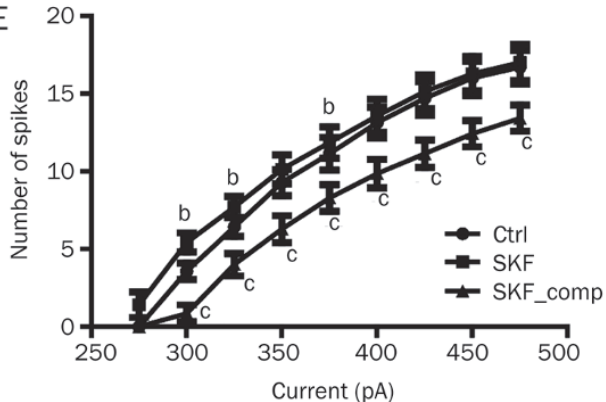

G

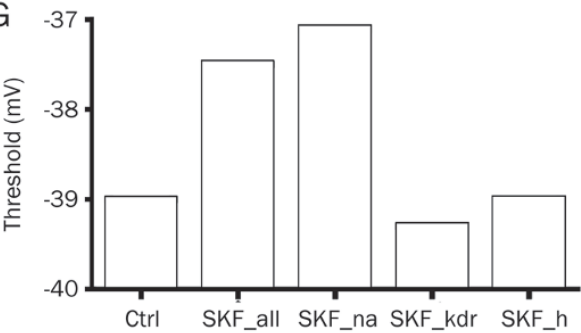

Figure 4. Effects of SKF83959 on excitability of the soma in the hippocampal pyramidal neuron in simulations and experiments. Soma excitability was measured by direct injection of a series of currents of different amplitudes at the soma. (A) In simulations, example traces show the voltage responses recorded at the soma in control and SKF83959 treated cells while modifying all three channels or one channel type alone with (right) or without (left) membrane potential compensation (all traces were obtained by injecting a current of $350 \mathrm{pA}$ for $500 \mathrm{~ms}$ ), as described in the methods. Spike numbers were detected under a series of current stimuli from 250 pA to 500 pA with steps of 25 pA with (C) or without (B) membrane potential compensation in simulations. (D) Example traces from experiments show the voltage responses recorded at the soma in control and SKF83959 (50 $\mu$ mol/L) treated cells during 75 pA current injection. (E) In experiments, spike numbers were detected under a series of current stimuli, from 25 pA to 225 pA, with steps of $25 \mathrm{pA}$ and with or without membrane potential compensation $(n=7)$. Rheobase current $(\mathrm{F})$ and spike threshold $(\mathrm{G})$ were examined as described in the methods using our mathematical neuronal model. Ctrl, control; SKF_all, modifying all three channels; SKF_na, modifying the Na ${ }^{+}$channel alone; SKF_kdr, modifying the kdr channel alone; SKF_h, modifying the HCN channel alone. SKF_all_comp, SKF_na_comp, SKF_kdr_comp and SKF_h_comp were the same as SKF_all, SKF_na, SKF_kdr, and SKF_h, except that the membrane potential was compensated to the control level; SKF, SKF83959 (50 $\mu \mathrm{mol} / \mathrm{L})$ treatment without membrane compensation; SKF_comp, SKF83959 (50 $\mu \mathrm{mol} / \mathrm{L})$ treatment with membrane potential compensation. ${ }^{\mathrm{b}} P<0.05$, ${ }^{\mathrm{c}} P<0.01$ vs control, paired $t$-test. 
A

a

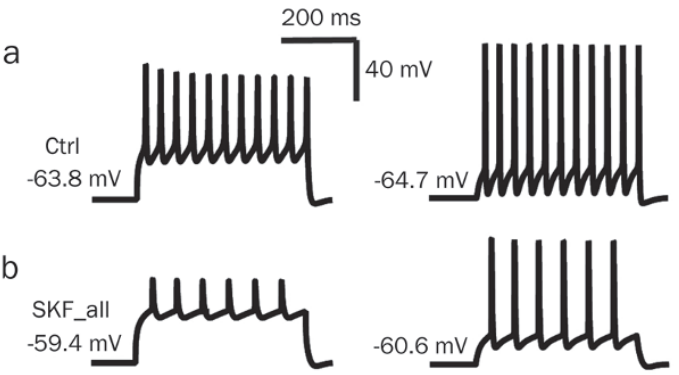

C

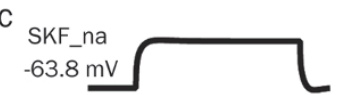

d

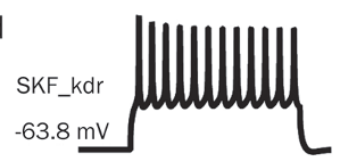

e

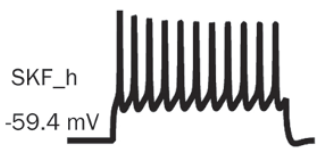

Dend
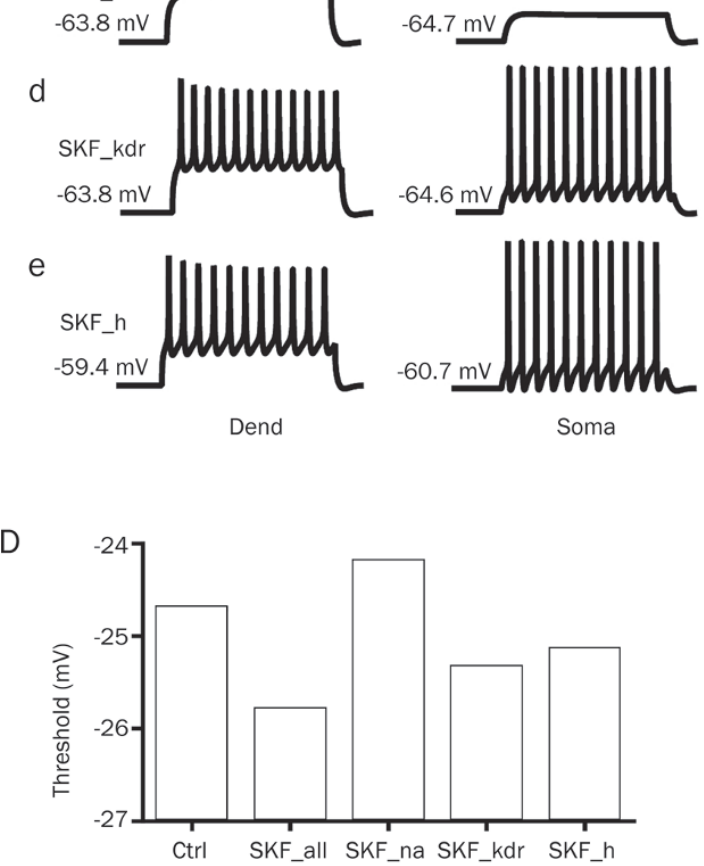

Soma
B
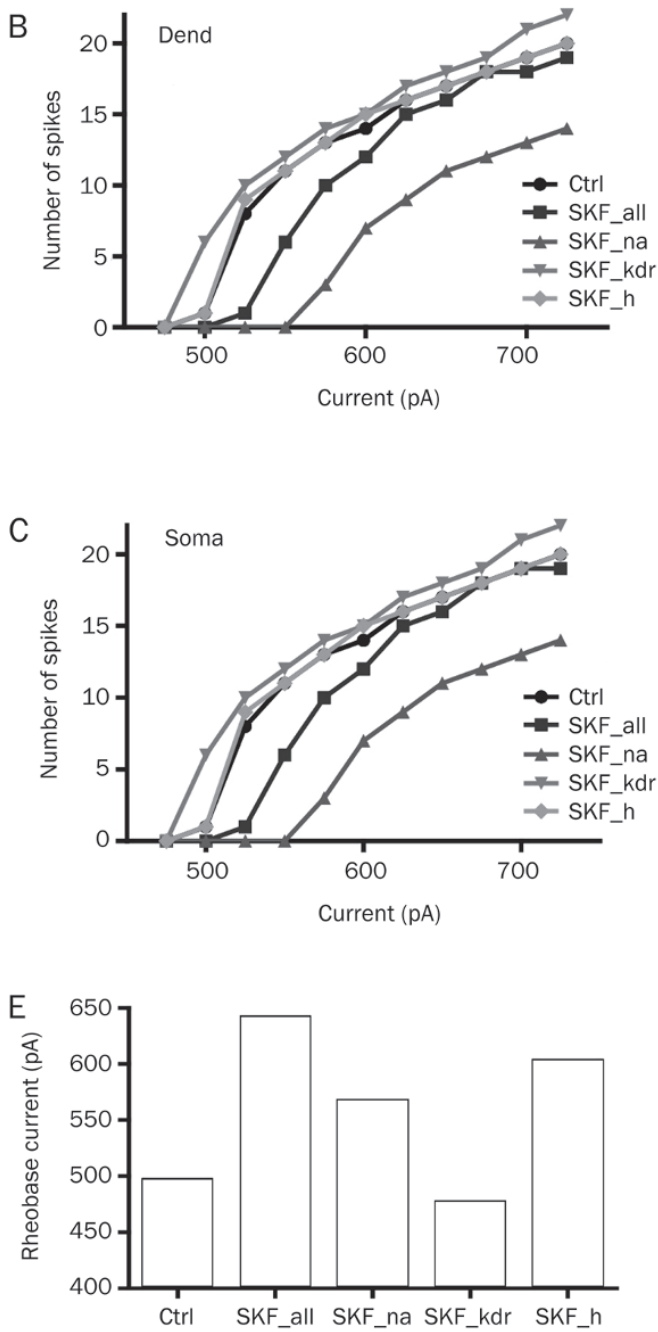

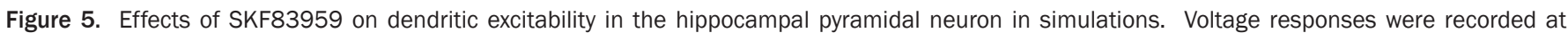

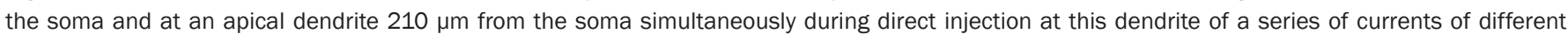

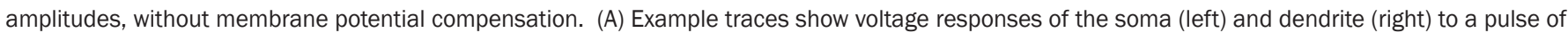

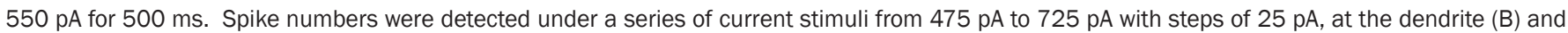

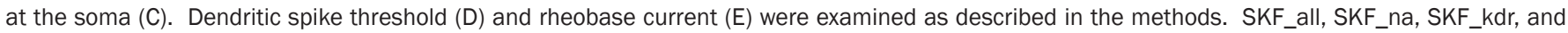
SKF_h are the same as in Figure 4.

the $\mathrm{HCN}$ channel resulted in a slight increase of the threshold (1.37 nA, Figure 6B). We further confirmed this result by experiments and we found that SKF83959 (50 $\mu \mathrm{mol} / \mathrm{L})$ significantly decreased the threshold for the induction of depolarization block $(885 \pm 43 \mathrm{pA}$ for control cells and $585 \pm 40 \mathrm{pA}$ for SKF83959 treated cells, $P<0.01$, Figure 6D).

Modeling of the effects of SKF83959 on EPSP temporal summation and synapse-driven firing in the hippocampal pyramidal neuron

Because the EPSP temporal summation along dendrites is very important in the induction of pyramidal neuronal firing ${ }^{[44]}$, and because many ion channels, including HCN channels, could affect this process ${ }^{[25,27-29]}$, we examined the effects of SKF83959 on EPSP temporal summation and consequently on neuronal firing, using our mathematical model. One synapse bearing both AMPA and NMDA mechanisms was placed on one apical dendrite ( $210 \mu \mathrm{m}$ from the soma). A train of 5 stimuli with the same interval between them were given to this synapse. By changing this interval, we could determine the pattern of EPSP temporal summation in response to stimuli with different frequencies. For sub-threshold EPSP temporal summation, $G_{\max }$ AMPA was set to $0.009 \mu \mathrm{S}$, which resulted in a $2.18 \mathrm{mV}$ amplitude for the first EPSP (Figure 7A \& 7B). SKF83959 treatment decreased the amplitude of the first EPSP to $1.92 \mathrm{mV}$ when membrane potential was not compensated to the control level (Figure 7A and 7B). This inhibition increased, with the amplitude of the first EPSP falling to $1.81 \mathrm{mV}$, when the membrane potential was compensated (Figure 7A). This effect appeared to be primarily attributable to the effect of SKF83959 on the HCN channel because modifying HCN channels alone decreased the amplitude to 
A
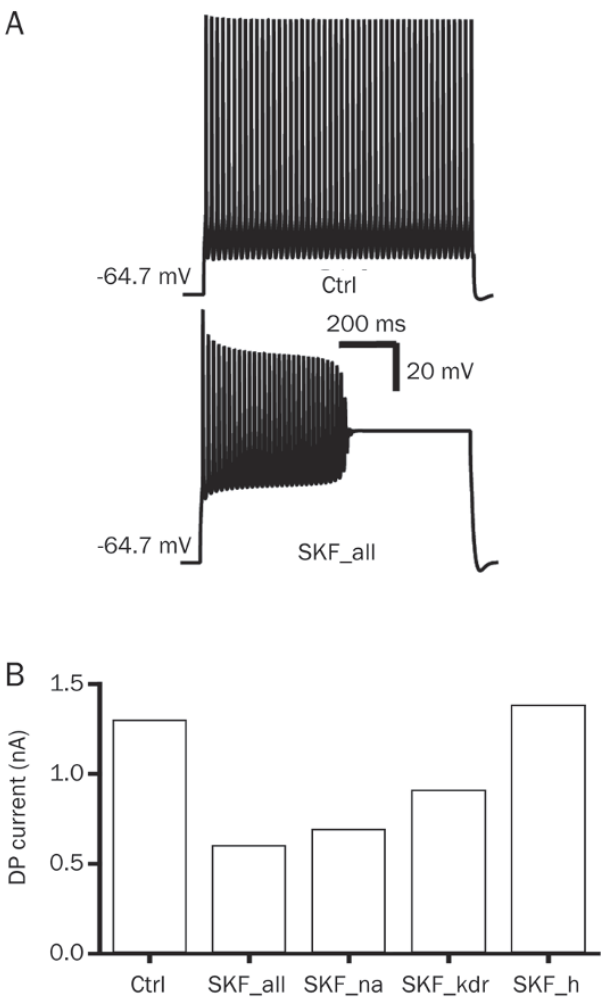

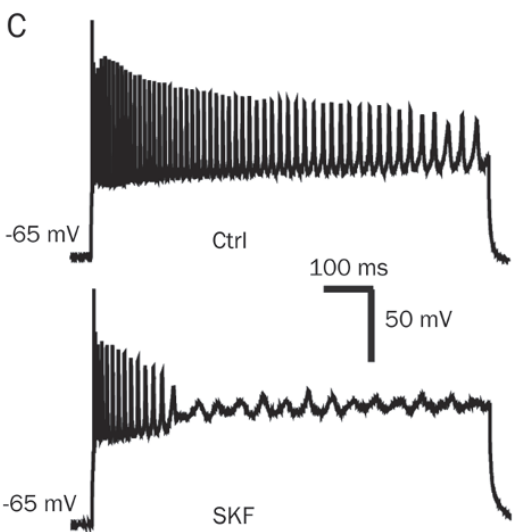

D

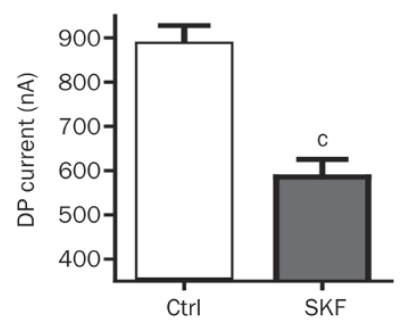

Figure 6. Effects of SKF83959 on depolarization block in the hippocampal pyramidal neuron in simulations and experiments. Depolarization block was examined as described in the methods with the membrane potential compensated to control levels. (A) In simulations, voltage responses at the soma with (bottom) or without (up) the overall effect of SKF83959 under $0.59 \mathrm{nA}$ current injection. (B) The minimal currents required to induce depolarization block under different conditions in simulations. (C) Experimental results showing voltage responses at the soma from experiments with (bottom) or without (up) SKF83959 (50 $\mu \mathrm{mol} / \mathrm{L})$ treatment during 800 pA current injection in simulations. (D) Experimental results showing the minimal currents required to induce depolarization block with or without SKF83959 (50 $\mathrm{mmol} / \mathrm{L})$ treatment $(n=7)$. SKF_all, SKF_na, SKF_kdr and SKF_h are the same as in Figure 4. Ctrl, control; SKF, SKF83959 $(50 \mu \mathrm{mol} / \mathrm{L})$ treatment. ${ }^{\mathrm{c}} P<0.01 \mathrm{vs}$ control, paired $t$-test.

$1.9 \mathrm{mV}$, whereas modifying $\mathrm{Na}^{+}$or $\mathrm{Kdr}$ channels resulted in only a slight change in amplitude (Figure 7B). A similar set of conditions was also applied to EPSP temporal summation. SKF83959 decreased EPSP temporal summation at all tested frequencies when membrane potential was not compensated (Figure 7C and 7D), and it decreased this summation further with membrane potential compensation (Figure 7C). The EPSP temporal summation ratio was 2.39 for control cells at $50 \mathrm{~Hz}$, and 1.59 and 2.16 for SKF83959 treated cells with or without membrane potential compensation, respectively (Figure 7C\&D). Again, the SKF83959-regulated HCN channel is the major factor in contributing to the observed difference in EPSP temporal summation with SKF83959 treatment (Figure 7D). To further examine how SKF83959 modulates the process of EPSP summation to induce action potentials (EPSP-spike coupling), we increased the $G_{\max }$ AMPA to a series of values at which the EPSP summated (at $20 \mathrm{~Hz}$ ) to the firing threshold. Consequently, we found that SKF83959 decreased the number of spikes elicited by EPSP temporal summation (Figure 7E and $7 \mathrm{~F}$ ) with the membrane potential uncompensated. An increase in the spike threshold due to the drug-modified $\mathrm{Na}^{+}$channel may contribute to this change because although SKF83959 may inhibit EPSP temporal summation, the exact membrane potential level for SKF83959 treated cells was still more depolarized than that for control cells (Figure 7A). For example, the membrane potentials at the fifth EPSP $(20 \mathrm{~Hz})$ were $-60.7 \mathrm{mV}$ and $-57.3 \mathrm{mV}$ for control and SKF83959 treated cells, respectively (Figure 7A).

To examine synapse-driven firing in a more physiologically relevant way, five independent synapses (with $G_{\max \_}$AMPA set to $0.021 \mu S$ ) were randomly placed on apical dendritic compartments and randomly and independently (Poisson) activated at different average frequencies (EPSP frequencies). SKF83959 was still able to decrease the firing frequency at all tested average EPSP frequencies, even when the depolarized membrane potential was left uncompensated (Figure 8). For example, SKF83959 decreased the firing frequency from $17.4 \pm 0.7 \mathrm{~Hz}$ to $2.4 \pm 0.4 \mathrm{~Hz}$ at an average EPSP frequency of 20 $\mathrm{Hz}$ (Figure 8). Modifying the $\mathrm{Na}^{+}$channel alone markedly decreased the firing frequency at every tested EPSP frequency, but modifying the $\mathrm{kdr}$ channel or $\mathrm{HCN}$ channel alone had a relatively small effect, which was consistent with the results shown in Figure 5 (Figure 8B). 

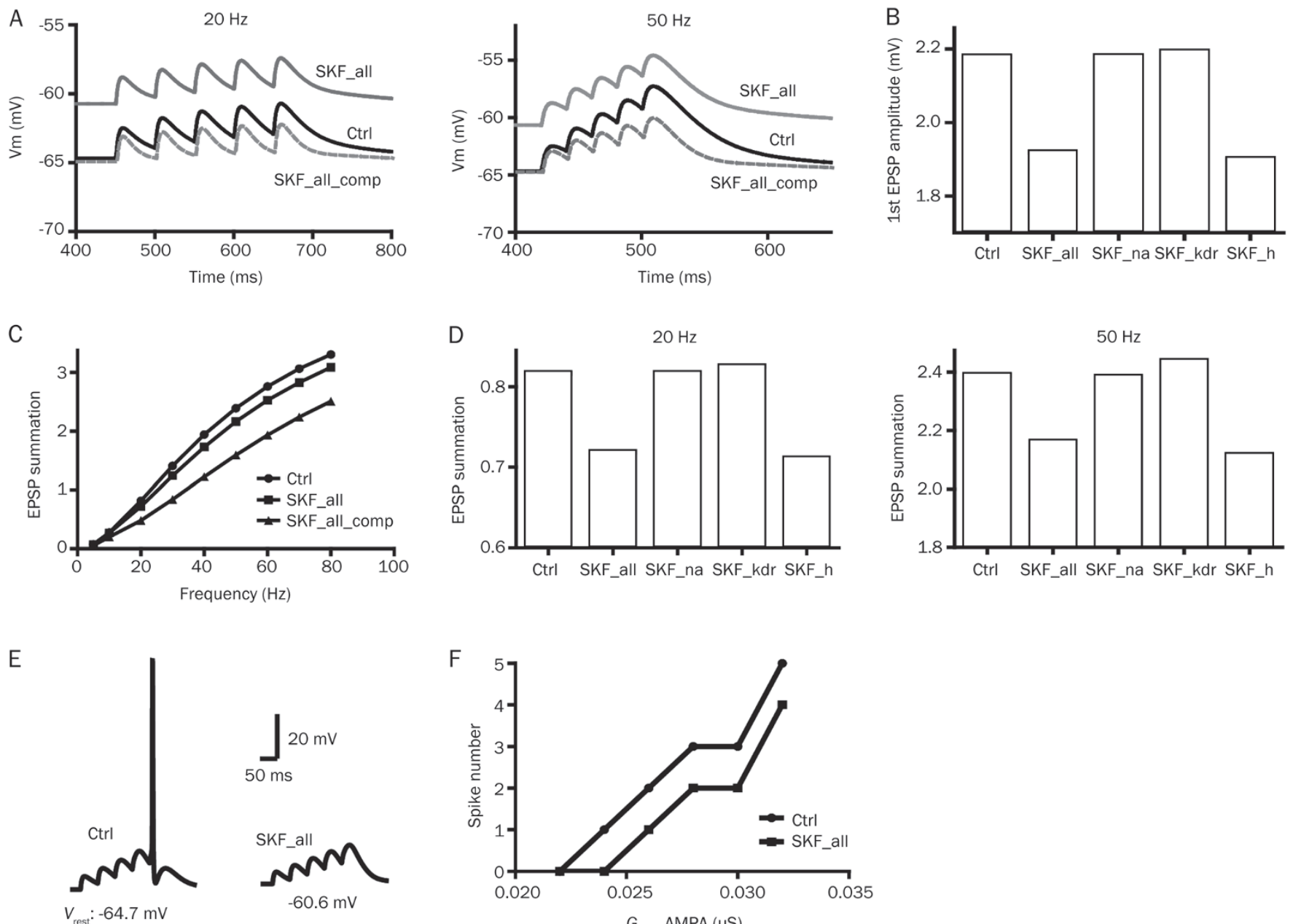

$-60.6 \mathrm{mV}$

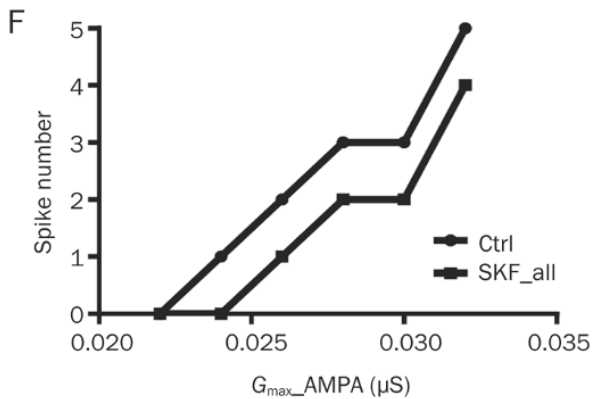

Figure 7. Effects of SKF83959 on EPSP temporal summation in simulations. EPSP temporal summation was measured as described in the methods. (A) Example traces of EPSP temporal summation during a train of 5 stimuli of either $20 \mathrm{~Hz}$ (left) or $50 \mathrm{~Hz}$ (right) with $\mathrm{G}_{\text {max }}$ AMPA set to $0.009 \mu$ S. EPSP temporal summations for control cells (solid black lines) and SKF83959 treated cells without (solid gray line) and with (dashed, gray line) membrane potential compensation. (B) The amplitude of the first EPSP without membrane potential compensation. (C) EPSP temporal summation ratio in response to a series of frequencies from $5 \mathrm{~Hz}$ to $80 \mathrm{~Hz}$ with or without membrane potential compensation. (D) EPSP temporal summation ratio in response to $20 \mathrm{~Hz}$ (left) and $50 \mathrm{~Hz}$ (right) stimulations under different conditions without membrane potential compensation. (E) Example traces showing EPSP-spike coupling without membrane potential compensation ( $G_{\text {max }}$ AMPA: $\left.0.024 \mu S\right)$. (F) Spike numbers in response to a series of values for $G_{\text {max }}$ AMPA under either control or SKF83959 treated conditions, without membrane potential compensation. SKF_all, SKF_all_comp, SKF_na, SKF_kdr, and SKF_h are the same as in Figure 4.

\section{Discussion}

In this study, we have characterized the effects of SKF83959 on the excitability of hippocampal pyramidal neurons using a realistic mathematical model of the hippocampal CA1 pyramidal neuron refined with experimental results obtained here and in previous studies ${ }^{[15-17]}$. We found that SKF83959 (50 $\mu \mathrm{mol} / \mathrm{L})$ shifted the $\mathrm{Na}^{+}$channel inactivation curve by $10.3 \mathrm{mV}$ but had no effect on the activation curve. We varied the time to recovery from inactivation of the $\mathrm{Na}^{+}$channel on a large scale (from 1/2 to 4 times of that of the control) in our model, but this change induced no significant effect on neuronal excitability. It should be noted that the slow inactivation component of the $\mathrm{Na}^{+}$channel was not considered in the current model; further work is needed to determine whether SKF83959 can affect the slow inactivation of the $\mathrm{Na}^{+}$ channel and how this effect could alter neuronal excitability because it has been demonstrated that slow inactivation in $\mathrm{Na}^{+}$channels is able to modulate neuronal excitability in the hippocampus $^{[45]}$. The simulated effects of SKF83959 on passive membrane properties were found to be in agreement with previously reported experimental results ${ }^{[15]}$. In addition, we found that SKF83959 differentially modulates excitability of the soma and dendritic cells. SKF83959 decreased excitability of the soma (investigated by current injection at the soma) only with the membrane potential compensated to the control level. However, SKF83959 decreased dendritic excitability (investigated by current injection at the dendrite) even without membrane potential compensation, causing a decrease in 
A
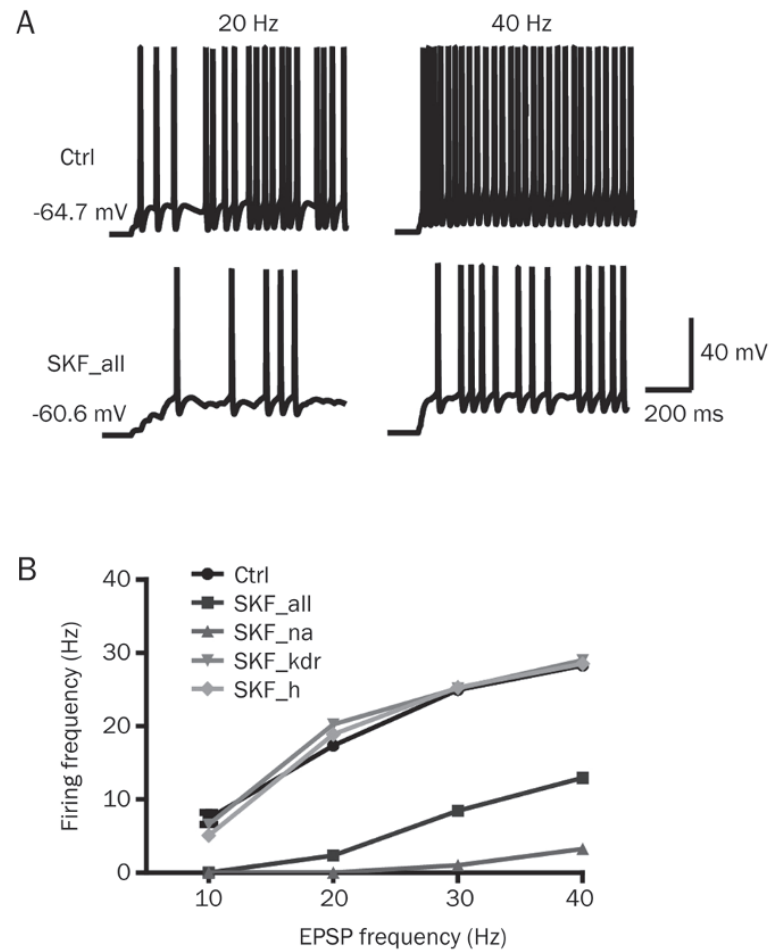

Figure 8. Effects of SKF83959 on synapse-driven firing in simulations of hippocampal pyramidal neurons. Five independent synapses (with $G_{\text {max }}$ AMPA set to $0.021 \mu \mathrm{S}$ ) were randomly placed on the apical dendritic compartments 150-400 $\mu \mathrm{m}$ from the soma and randomly (Poisson) activated at different average frequencies (EPSP frequencies). (A) Example traces showing the voltage response at the soma to average EPSP frequencies of $20 \mathrm{~Hz}$ (left) and $40 \mathrm{~Hz}$ (right) without membrane potential compensation. (B) The firing frequencies in response to a series of average EPSP frequencies, from $10 \mathrm{~Hz}$ to $40 \mathrm{~Hz}$, under either control or SKF83959 treated conditions, without membrane potential compensation. SKF_all, SKF_na, SKF_kdr, and SKF_h are the same as in Figure 4.

the number of spikes at the soma. In contrast, SKF83959 facilitated depolarization block in pyramidal neurons. Finally, we found that SKF83959 reduced EPSP temporal summation and the synapse-driven firing frequency.

To overcome the space-clamp problem ${ }^{[46]}$ and to minimize the effects of synaptic transmission, we measured the gating kinetics of $\mathrm{Na}^{+}$channels in dissociated hippocampal neurons prepared from newborn (age 3-7 d) Sprague-Dawley rats. To expand upon previous experimental results ${ }^{[15]}$ and to test the predictions of our morphologically realistic neuronal model, we measured excitability and depolarization block at the soma in brain slices from Sprague-Dawley rats (age 2-3 weeks); in these slices, the shapes of the pyramidal neurons retained relatively intact. Because CNQX and picrotoxin were added to solutions to block synaptic transmission, and because, to our knowledge, there is no significant difference between these two developmental stages in terms of the properties of ion channels relevant to our current work, we are able to rule out the possibility of inconsistencies between these data.
We previously found that SKF83959 regulated multiple ion channel activities, including those of $\mathrm{Na}^{+}, \mathrm{kdr}$, and $\mathrm{HCN}$ channels ${ }^{[15-17]}$, but the specific contributions of the effects of SKF83959 on individual ion channels to neuronal excitability were not clear. In the present study, we demonstrated that modifying the $\mathrm{Na}^{+}$channel alone increases the rheobase current and consequently results in decreased excitability at both the soma and the dendrite, in agreement with previous reports ${ }^{[18-20]}$. In contrast with $\mathrm{Na}^{+}$channels, modifying the $\mathrm{kdr}$ channel alone can lead to a slight increase in neuronal excitability. However, the specific role of HCN channels in SKF83959-regulated neuronal excitability is rather complex. It has been reported that the HCN channel can produce either excitatory (depolarizing the membrane) or inhibitory (decreasing the input resistance) effects on the excitability of pyramidal neurons ${ }^{[25-28]}$. In previous studies, the excitability of these neurons has been measured with the depolarized membrane potential compensated to the control level, which in some respects resulted in selectively neglecting the excitatory effect. On the other hand, the gradient distribution of HCN channels in the soma and in the dendrite should also be considered ${ }^{[27]}$. In the present study, we examined the effects of SKF83959 in modulating HCN channels with or without membrane potential compensation. In the soma, modifying $\mathrm{HCN}$ channels alone slightly increased excitability without membrane potential compensation, which indicates that the HCN channel plays an excitatory role in the soma. In dendrites, modifying the HCN channel alone had little effect on SKF83959-regulated neuronal excitability, which may be the result of a balance between excitatory and inhibitory effects. The difference between the responses of the soma and dendrites was most likely due to the much higher density of HCN channels in dendrites than in soma ${ }^{[27,37]}$. Moreover, we found that modifying the $\mathrm{Na}^{+}$channel or $\mathrm{kdr}$ channel alone facilitated depolarization block, consistent with previous studies ${ }^{[31]}$. In contrast, modifying the $\mathrm{HCN}$ channel alone increased the depolarization block threshold, which is likely attributable to the decrease in the input resistance.

It has been shown that the HCN channel exerts a strong regulatory force on EPSP temporal summation along the dendrite because of its high density and because of shunting effects ${ }^{[25,27-29]}$. In the present study, modifying the $\mathrm{HCN}$ channel alone inhibited EPSP temporal summation, but the membrane at the last EPSP was nonetheless more depolarized in SKF83959 treated cells than in control cells. This effect of increasing HCN current on EPSP temporal summation and membrane potentials that we observed differs from the results in a previous report ${ }^{[28]}$. In that study, the authors noted that when the amplitude of EPSPs were large enough, at the last EPSP, the membrane was more hyperpolarized with $\mathrm{HCN}$ channels than without HCN channels because of the opening of M-type potassium channels ${ }^{[28]}$. In the present study we did not include M-type potassium channels. Even so, in combination with the effects on $\mathrm{Na}^{+}$channels, SKF83959 could still decrease the synapse-driven firing frequency, either in EPSP-spike coupling simulations or in simulations with more 
synapses being activated randomly. Future work in which an M-type potassium channel mechanism is inserted into the model would be necessary to confirm our hypothesis.

Physiologically, neurons are activated by the summation of multiple EPSPs that originate at dendrites. Thus, we examined the effects of SKF83959 on neuronal excitability using the model neuron activated by 5 synapses randomly placed on the dendrites. Our results revealed how the effects of SKF83959 on both the intrinsic properties of the neuron and on EPSP temporal summation combined to modulate neuronal firing. SKF83959 decreased the synapse-driven firing frequency even without the membrane potential compensated to control levels, which further supports the conclusions drawn from our results on neuronal excitability during current injection.

SKF83959, unlike other D1 receptor agonists such as SKF38393 and SKF83822, which exert their function primarily via the D1-like receptor-mediated signaling pathway ${ }^{[47-49]}$, elicits many biological responses that are either dependent or independent of D1-like receptors ${ }^{[5-7]}$. SKF83959 has been shown to produce potent anti-parkinsonian effects in primate and rodent model ${ }^{[5-7]}$, and chronic administration of SKF83959 also attenuates the development of L-DOPA-induced dyskinesia (LID) in 6-OH-DOPA-lesioned rat models; this latter result may be associated with the drug's neuroprotective effects ${ }^{[5,8]}$. We have previously reported that SKF83959 has potent neuroprotective effects that are only partially attributable to D1-like receptor activation ${ }^{[10]}$. It is conceivable that a D1-receptorindependent mechanism, such as SKF83959-mediated inhibition of neuronal excitability or inhibition of the kdr channel, may also contribute to these neuroprotective effects ${ }^{[50,51]}$. In addition, it has been documented that the excitability of striatal neurons is increased in the 6-OH-DOPA-lesioned rat model for Parkinson's disease ${ }^{[12-14]}$. Inhibition of neuronal excitability may partially contribute to the drug's anti-Parkinsonian effects.

Abnormal neuronal excitability plays a fundamental role in the pathophysiology of epilepsy ${ }^{[32,33]}$. Depolarization block is proposed to be associated with the initiation and spread of focal epileptic seizures that generates a paroxysmal depolarizing effect in the involved neurons, which may cause the inactivation of $\mathrm{Na}^{+}$channels ${ }^{[31-33,52]}$. In our study, the effects of SKF83959 on $\mathrm{Na}^{+}$channel density and on the $\mathrm{Na}^{+}$channel inactivation curve facilitated depolarization block in hippocampal pyramidal neurons. Moreover, although inhibition of $\mathrm{Na}^{+}$channels is one of the most common activities of many antiepileptic drugs ${ }^{[53]}$, the importance of the HCN channel has recently also been proposed ${ }^{[1,54,55]}$. That SKF83959 decreases excitability and facilitates depolarization block in hippocampal pyramidal neurons could indicate that the drug may have a potential antiepileptic effect. In fact, the pharmacological effects of SKF83959, including $\mathrm{Na}^{+}$channel inhibition and shifting of the voltage dependent activation of the HCN channel, are very similar to those of the widely used antiepileptic drug lamotrigine ${ }^{[37,56]}$. In this regard, SKF83959 not only shifted the activation curve of the $\mathrm{HCN}$ channel but also increased the maximum conductance of this channel, as shown by the increased amplitude of current, even at $-120 \mathrm{mV}^{[15]}$. The potential antiepileptic effect of SKF83959 is currently under investigation.

Taken together, the effects of SKF83959 result in a decrease in neuronal excitability that may at least partially contributes to the drug's neuroprotective and anti-parkinsonian effects. The effects of SKF83959 also suggest that it may have a potential antiepileptic effect.

\section{Acknowledgements}

This work was financially supported by grants from the National Natural Science Foundation of China (81130023, 31373382, and 31271143) and the National Basic Research Plan (973) of the Ministry of Science and Technology of China (2011CB5C4403). We are also grateful for support from the Priority Academic Program Development of Jiangsu Higher Education Institutes (PAPD).

\section{Author contribution}

Shang-lin ZHOU, Hong-yuan CHU, and Xue-chu ZHEN designed the study; Shang-lin ZHOU and Hong-yuan CHU performed the experiments; Shang-lin ZHOU did the simulations; Shang-lin ZHOU, Guo-zhang JIN, Jian-min CUI, and Xue-chu ZHEN wrote the manuscript.

\section{References}

1 Jin LQ, Goswami S, Cai G, Zhen X, Friedman E. SKF83959 selectively regulates phosphatidylinositol-linked D1 dopamine receptors in rat brain. J Neurochem 2003; 85: 378-86.

2 Panchalingam S, Undie AS. SKF83959 exhibits biochemical agonism by stimulating $\left[{ }^{35} \mathrm{~S}\right] \mathrm{GTP}$ gamma $\mathrm{S}$ binding and phosphoinositide hydrolysis in rat and monkey brain. Neuropharmacology 2001; 40: 826-37.

3 Arnt J, Hyttel J, Sanchez C. Partial and full dopamine D1 receptor agonists in mice and rats: relation between behavioural effects and stimulation of adenylate cyclase activity in vitro. Eur J Pharmacol 1992; 213: 259-67.

4 Fang X, Guo L, Jia J, Jin GZ, Zhao B, Zheng YY, et al. SKF83959 is a novel triple reuptake inhibitor that elicits anti-depressant activity. Acta Pharmacol Sin 2013; 34: 1149-55.

5 Gnanalingham KK, Erol DD, Hunter AJ, Smith LA, Jenner P, Marsden CD. Differential anti-parkinsonian effects of benzazepine D1 dopamine agonists with varying efficacies in the MPTP-treated common marmoset. Psychopharmacology (Berl) 1995; 117: 275-86.

6 Zhen X, Goswami S, Friedman E. The role of the phosphatidyinositollinked D1 dopamine receptor in the pharmacology of SKF83959. Pharmacol Biochem Behav 2005; 80: 597-601.

7 Andringa G, Stoof JC, Cools AR. Sub-chronic administration of the dopamine D-1 antagonist SKF 83959 in bilaterally MPTPtreated rhesus monkeys: stable therapeutic effects and wearing-off dyskinesia. Psychopharmacology 1999; 146: 328-34.

8 Zhang H, Ma L, Wang F, Chen J, Zhen X. Chronic SKF83959 induced less severe dyskinesia and attenuated L-DOPA-induced dyskinesia in 6-OHDA-lesioned rat model of Parkinson's disease. Neuropharmacology 2007; 53: 125-33.

9 Guo L, Zhao JH, Jin GZ, Zhao B, Wang GH, Zhang A, et al. SKF83959 is a potent allosteric modulator of sigma-1 receptor. Mol Pharmacol 2013; 83: 577-86. 
10 Yu Y, Wang JR, Sun PH, Guo Y, Zhang ZJ, Jin GZ, et al. Neuroprotective effects of atypical D1 receptor agonist SKF83959 are mediated via D1 receptor-dependent inhibition of glycogen synthase kinase-3 beta and a receptor-independent anti-oxidative action. J Neurochem 2008; 104: 946-56.

11 Shah MM, Anderson AE, Leung V, Lin XD, Johnston D. Seizure-induced plasticity of $h$ channels in entorhinal cortical layer III pyramidal neurons. Neuron 2004; 44: 495-508.

12 Chen MT, Morales M, Woodward DJ, Hoffer BJ, Janak PH. In vivo extracellular recording of striatal neurons in the awake rat following unilateral 6-hydroxydopamine lesions. Exp Neurol 2001; 171: 72-83.

13 Schultz W, Ungerstedt U. Short-term increase and long-term reversion of striatal cell activity after degeneration of the nigrostriatal dopamine system. Exp Brain Res 1978; 33: 159-71.

14 Kish $\sqcup$, Palmer MR, Gerhardt GA. Multiple single-unit recordings in the striatum of freely moving animals: effects of apomorphine and D-amphetamine in normal and unilateral 6-hydroxydopamine-lesioned rats. Brain Res 1999; 833: 58-70.

$15 \mathrm{Chu}$ HY, Gu QH, Jin GZ, Hu GY, Zhen XC. Electrophysiological effects of SKF83959 on hippocampal CA1 pyramidal neurons: potential mechanisms for the drug's neuroprotective effects. Plos One 2010; 5: pii: e13118. doi: 10.1371/journal.pone.0013118.

16 Chen XQ, Zhang J, Neumeyer JL, Jin GZ, Hu GY, Zhang A, et al. Arylbenzazepines are potent modulators for the delayed rectifier $\mathrm{K}^{+}$ channel: a potential mechanism for their neuroprotective effects. Plos One 2009; 4: e5811.

17 Chu HY, Wu QQ, Zhou SL, Cao XH, Zhang A, Jin GZ, et al. SKF83959 suppresses excitatory synaptic transmission in rat hippocampus via a dopamine receptor-independent mechanism. J Neurosci Res 2011; 89: 1259-66.

18 Martina M, Jonas P. Functional differences in $\mathrm{Na}^{+}$channel gating between fast-spiking interneurones and principal neurones of rat hippocampus. J Physiol (London) 1997; 505: 593-603.

19 Raman IM, Bean BP. Resurgent sodium current and action potential formation in dissociated cerebellar Purkinje neurons. J Neurosci 1997; 17: 4517-26.

20 Stuart G, Spruston N, Sakmann B, Hausser M. Action potential initiation and backpropagation in neurons of the mammalian CNS. Trends Neurosci 1997; 20: 125-31.

21 Du J, Haak LL, Phillips-Tansey E, Russell JT, McBain CJ. Frequencydependent regulation of rat hippocampal somato-dendritic excitability by the $\mathrm{K}^{+}$channel subunit Kv2.1. J Physiol 2000; 522: 19-31.

22 Kang J, Huguenard JR, Prince DA. Voltage-gated potassium channels activated during action potentials in layer $\mathrm{V}$ neocortical pyramidal neurons. J Neurophysiol 2000; 83: 70-80.

23 Malin SA, Nerbonne JM. Delayed rectifier $\mathrm{K}^{+}$currents, I-K, are encoded by Kv2 alpha-subunits and regulate tonic firing in mammalian sympathetic neurons. J Neurosci 2002; 22: 10094-105.

24 Mohapatra DP, Misonou H, Pan SJ, Held JE, Surmeier DJ, Trimmer JS. Regulation of intrinsic excitability in hippocampal neurons by activitydependent modulation of the $\mathrm{K}(\mathrm{V}) 2.1$ potassium channel. Channels 2009; 3: 46-56.

25 Bean BP. Inhibition by an excitatory conductance: a paradox explained. Nat Neurosci 2009; 12: 530-2.

26 Kase D, Imoto K. The role of HCN channels on membrane excitability in the nervous system. J Signal Transduct 2012; 2012: 619747.

27 Wahl-Schott C, Biel M. HCN channels: structure, cellular regulation and physiological function. Cell Mol Life Sci 2009; 66: 470-94.

28 George MS, Abbott LF, Siegelbaum SA. HCN hyperpolarizationactivated cation channels inhibit EPSPs by interactions with M-type $\mathrm{K}^{+}$ channels. Nat Neurosci 2009; 12: 577-84.
29 Magee JC. Dendritic I-h normalizes temporal summation in hippocampal CA1 neurons. Nat Neurosci 1999; 2: 508-14.

30 Richards CD, Shiroyama T, Kitai ST. Electrophysiological and immunocytochemical characterization of GABA and dopamine neurons in the substantia nigra of the rat. Neuroscience 1997; 80: 545-57.

31 Bianchi D, Marasco A, Limongiello A, Marchetti C, Marie H, Tirozzi $B$, et al. On the mechanisms underlying the depolarization block in the spiking dynamics of CA1 pyramidal neurons. J Comput Neurosci 2012; 33: 207-25.

32 McCormick DA, Contreras D. On the cellular and network bases of epileptic seizures. Annu Rev Physiol 2001; 63: 815-46.

33 Dichter MA, Ayala GF. Cellular mechanisms of epilepsy - a statusreport. Science 1987; 237: 157-64.

34 Grace AA, Bunney BS, Moore H, Todd CL. Dopamine-cell depolarization block as a model for the therapeutic actions of antipsychotic drugs. Trends Neurosci 1997; 20: 31-7.

35 Kuznetsova AY, Huertas MA, Kuznetsov AS, Paladini CA, Canavier CC. Regulation of firing frequency in a computational model of a midbrain dopaminergic neuron. J Comput Neurosci 2010; 28: 389-403.

36 Tucker KR, Huertas MA, Horn JP, Canavier CC, Levitan ES. Pacemaker Rate and depolarization block in nigral dopamine neurons: a somatic sodium channel balancing act. J Neurosci 2012; 32: 14519-31.

37 Poolos NP, Migliore M, Johnston D. Pharmacological upregulation of h-channels reduces the excitability of pyramidal neuron dendrites. Nat Neurosci 2002; 5: 767-74.

38 Gao ZB, Chen XQ, Hu GY. Inhibition of excitatory synaptic transmission by trans-resveratrol in rat hippocampus. Brain Res 2006; 1111: 41-7.

39 Hines ML, Carnevale NT. The NEURON simulation environment. Neural Comput 1997; 9: 1179-209.

40 Migliore M, Hoffman DA, Magee JC, Johnston D. Role of an A-type $\mathrm{K}^{+}$conductance in the back-propagation of action potentials in the dendrites of hippocampal pyramidal neurons. J Comput Neurosci 1999; 7: 5-15.

41 Durstewitz D, Seamans JK, Sejnowski TJ. Dopamine-mediated stabilization of delay-period activity in a network model of prefrontal cortex. J Neurophysiol 2000; 83: 1733-50.

42 Cutsuridis V, Cobb S, Graham BP. Encoding and retrieval in a model of the hippocampal CA1 microcircuit. Hippocampus 2010; 20: 423-46.

43 Ferrante M, Blackwell KT, Migliore M, Ascoli GA. Computational Models of neuronal biophysics and the characterization of potential neuropharmacological targets. Curr Med Chem 2008; 15: 2456-71.

44 Spruston N. Pyramidal neurons: dendritic structure and synaptic integration. Nat Rev Neurosci 2008; 9: 206-21.

45 Park YY, Johnston D, Gray R. Slowly inactivating component of $\mathrm{Na}^{+}$ current in peri-somatic region of hippocampal CA1 pyramidal neurons. J Neurophysiol 2013; 109: 1378-90.

46 Armstrong CM, Gilly WF. Access resistance and space clamp problems associated with whole-cell patch clamping. Methods Enzymol 1992; 207: 100-22.

47 O'Sullivan GJ, Roth BL, Kinsella A, Waddington JL. SK\&F 83822 distinguishes adenylyl cyclase from phospholipase C-coupled dopamine D1-like receptors: behavioural topography. Eur J Pharmacol 2004; 486: $273-80$.

48 Seeman P, Vantol HHM. Dopamine-receptor pharmacology. Trends Pharmacol Sci 1994; 15: 264-70.

49 Sibley DR, Leff SE, Creese I. Interactions of novel dopaminergic ligands with D-1 and D-2 dopamine-receptors. Life Sci 1982; 31: 637-45.

50 Yu SP, Farhangrazi ZS, Ying HS, Yeh CH, Choi DW. Enhancement of outward potassium current may participate in beta-amyloid peptide- 
induced cortical neuronal death. Neurobiol Disease 1998; 5: 81-8.

51 Pal S, Hartnett KA, Nerbonne JM, Levitan ES, Aizenman E. Mediation of neuronal apoptosis by Kv2.1-encoded potassium channels. J Neurosci 2003; 23: 4798-802.

52 Ayala GF, Dichter M, Gumnit RJ, Matsumot.H, Spencer WA. Genesis of epileptic interictal spikes - new knowledge of cortical feedbacksystems suggests a neurophysiological explanation of brief paroxysms. Brain Res 1973; 52: 1-17.

53 Czapinski P, Blaszczyk B, Czuczwar SJ. Mechanisms of action of antiepileptic drugs. Curr Top Med Chem 2005; 5: 3-14.

54 Poolos NP. HCN channelopathy in epilepsy. Epilepsia 2010; 51 (Suppl 5): 12.

55 Shah MM, Huang Z, Martinello K. HCN and K(V)7 (M-) channels as targets for epilepsy treatment. Neuropharmacology 2013; 69: 75-81.

56 Kuo CC, Lu L. Characterization of lamotrigine inhibition of $\mathrm{Na}^{+}$ channels in rat hippocampal neurones. Br J Pharmacol 1997; 121: 1231-8. 